\title{
Opto-Physiological Modeling Applied to Photoplethysmographic Cardiovascular Assessment
}

\author{
Sijung Hu*, Vicente Azorin-Peris and Jia Zheng \\ School of Electronic, Electrical and Systems Engineering, Loughborough University, \\ Loughborough Leicestershire LE11 3TU, UK
}

Submitted September, 2012. Accepted for publication June 2013.

\begin{abstract}
This paper presents opto-physiological (OP) modeling and its application in cardiovascular assessment techniques based on photoplethysmography (PPG). Existing contact point measurement techniques, i.e., pulse oximetry probes, are compared with the next generation noncontact and imaging implementations, i.e., non-contact reflection and camera-based PPG. The further development of effective physiological monitoring techniques relies on novel approaches to OP modeling that can better inform the design and development of sensing hardware and applicable signal processing procedures. With the help of finite-element optical simulation, fundamental research into OP modeling of photoplethysmography is being exploited towards the development of engineering solutions for practical biomedical systems. This paper reviews a body of research comprising two OP models that have led to significant progress in the design of transmission mode pulse oximetry probes, and approaches to 3D blood perfusion mapping for the interpretation of cardiovascular performance.
\end{abstract}

Keywords: opto-physiological modeling, pulse oximetry, imaging photoplethysmography (iPPG), region of interest (ROI), signal and image processing, cardiovascular assessment

\section{INTRODUCTION}

Photoplethysmography (PPG) is an optical technique for non-invasive measurement of changes in blood volume, indirectly based on variations in light intensity passing through, or reflected from, skin tissue, through the use of an illumination source and a photodetector [1]. Variations in the detected output originate from the pulsation of arterial blood within the peripheral vasculature as stimulated by the quasi-periodic cardiac cycle [2].

PPG is ideally suited for the assessment of peripheral circulation in medical and biomedical monitoring setups [5, 6]. Prior art defines that PPG signals are composed of two key components [7]: a pulsatile part (AC component) of total absorbance from both

*Corresponding author: Sijung Hu, Leader of Photonics Engineering and Health Technology Research Group, School of Electronic, Electrical and Systems Engineering, Loughborough University, Ashby Road, Loughborough Leicestershire LE11 3TU, UK. Phone: +44 (0) 1509 227058, +44 (0) 7843566982 (mobile), Fax: +44 (0) 1509 227014. E-mail: S.Hu@Iboro.ac.uk Other authors: V.Azorin-Peris@lboro.ac.uk; jiacandy@hotmail.com. 
arterial blood and venous blood, and a quasi-static non-pulsatile component (DC component) attributed to tissue and venous blood. The AC component of the PPG signal has been used to assess peripheral vascular compliance [8], and the DC component of the PPG signal can be affected by respiration, the sympathetic nervous system and thermoregulation [3]. The normalized pulse wave using both AC and DC components could indicate peripheral blood volume changes more reliably [10].

Among PPG applications, pulse oximetry-the determination of arterial oxygen saturation-is the most widespread, thanks to its ability to alert the clinician of the presence of hypoxemia in real time [11]. A non-invasive method of monitoring peripheral venous oxygen saturation $(\mathrm{SxvO} 2)$ with an artificial venous pulse at the finger was successfully developed to serve as an adjunct/early warning indicator of imbalances in oxygen supply and demand [12]. Pulse oximetry is an important tool to evaluate and monitor pulmonary diseases, but the current knowledge of arterial oxygen saturation $\left(\mathrm{SpO}_{2}\right)$ values provides a limited description of the distribution of low pulse oximetry values in a general adult population and their association with certain predictors [13]. Pulse oximetry data such as saturation of peripheral oxygen ( $\mathrm{SpO} 2)$ and pulse rate are vital signals for early diagnosis of cardiovascular disease, and such a system should be equipped with communication capabilities to achieve remote monitoring and management [14].

\subsection{Limitations of PPG Technology}

Despite their proven usefulness, oximeters derived from photoplethysmography have a number of factors that lead to inaccurate readings and limit their applicability. The contact PPG (cPPG) probes most commonly found in commercially available pulse oximeters use only two wavelengths - an arrangement that assumes the presence of only hemoglobin and oxyhemoglobin in the blood. The presence of other species of hemoglobin in blood, i.e. carboxyhemoglobin and methemoglobin [15], and other factors, i.e., heavy skin pigmentation [16], have been proven to lead to measurement errors in pulse oximetry. The following are potential areas of research to increase the reliability and applicability of the oximeter technology:

(1) Commercial pulse oximeters convert the ratio of normalized pulsatile components into an equivalent arterial oxygen saturation by means of a linear or bilinear relationship look-up table which is determined by comparison of oximeter readings with invasive blood gas measurements [17]. This calibration is generally applied with oxygen saturation values above $70 \%$ due to serious health risks encountered by test subjects for saturations bel ow this point. Even though this factor does not affect the performance of oximeters in their primary function of warning clinicians of an unhealthy condition, it does limit their applicability, for instance, in the assessment of fetal wellbeing [18].

(2) A common source of PPG signal corruption is the inadvertent measurement of ambient light and voluntary or involuntary movement of the patient [19]. Ambient light artifact is generally not an issue since oximeters are designed to isolate its contribution to the signal, and the effect can be further reduced by covering the probe with an opaque shield when necessary [20]. Motion artifact, however, is a 
complex issue due to its contribution to the PPG signal being several orders of magnitude larger than that of the arterial pulsations, and to the spectral and temporal dependence between the arterial pulsations and the motion artifacts [21].

(3) In cases of low perfusion such as in critically ill patients, the low signal-to-noise ratio (SNR) encountered under a lack of a strong pulsatile PPG signal component leads to inaccurate readings [22]. This poses a serious drawback, as an adequate knowledge of a patient's oxygenation is especially important under these circumstances.

(4) Skin pigmentation and nail polish absorb red and infrared (IR) light at differing degrees, an effect which can reduce the SNR and cause minor offsets in oximeter readings [23]. Interference from certain substances in blood, however, can severely compromise the reliability of saturation readings. An increased level of dyshemoglobins in blood, such as carboxyhemoglobin and methemoglobin, are known to cause inaccurate oximetry readings due to the fact that commercial device calibration is performed under healthy volunteers with normal levels of these dyshemoglobins [24]. Intravenous dyes absorb light from the two wavelengths at different degrees, and thus manifest as a change in the absorption characteristics of the affected blood volume, rendering device calibration curves ineffective [20].

\subsection{Progress in PPG Technology}

Motivated by general trends towards remote sensing and the desire to reduce physical restrictions and cabling associated with patient monitoring, research efforts are targeting non-contact camera-based PPG [25, 26, 27]. An imaging PPG (iPPG) system can result in an alternative functional imaging solution to clinical situations that are currently addressed utilizing magnetic resonance imaging (MRI) and laser Doppler perfusion imaging (LDPI). This technique brings new insights by providing hemodynamic imaging and mapping capabilities. The functionality of camera-based iPPG has been well demonstrated through the visualization of blood perfusion [27, 28, 29], where the simultaneous capture of PPG waveforms from a region of interest on the extremities under illumination at two wavelengths $(660 \mathrm{~nm}$ and $880 \mathrm{~nm})$ was achieved. The 3-D mapping of skin blood microcirculation [28] was constructed based upon the arterial pulsation extracted from the iPPG signals, and a previous study shows the comparability of iPPG with conventional cPPG and the capability of obtaining good quality PPG pulsatile signals from a human face [29]. The recent proliferation of mobile applications is bringing about an inevitable trend in the collection of physiological parameters for personal healthcare $[30,31,32,33]$. In a growing list of personal physiological monitoring apps available online for the iPhone, iPad, and Android devices, 'Instant Heart Rate' by Azumio [34] and 'Vital Signs Camera' by Philips [35] have attracted significant public interest despite not being intended for clinical or diagnostic use.

The success of solutions to engineering challenges in PPG technology depends heavily on the validity of the assumptions employed to interpret the PPG signals. In OP models based on the Beer-Lambert equation, the measuring site is treated as a bloodfilled cuvette with no scattering effects and the light sources are assumed to be monochromatic [36, 37]. With the increasing availability and accuracy of tissue optical 
properties [38, 39, 40], the use of numerical solutions of light propagation in human tissue are providing increasingly valuable insights into such mechanisms. Motion artifact is one of key challenges in PPG-based biomedical monitoring techniques, and the reduction of this phenomenon has been investigated through different approaches $[41,42]$ to improve the SNR and to obtain a more robust data readout for more reliable clinical assessment.

The long-term goal of the body of research presented in this paper is to validate increasingly accurate and applicable models of opto-physiological systems. This paper presents three aspects of the development of effective OP models: 1) modeling of transmission- and reflection-mode PPG, 2) empirical studies and their signal and image processing procedures, and 3) the applicability of OP models in physiological monitoring systems.

\section{MODELING AND SIMULATION}

The concepts underlying OP modeling are considered in the context of transmissionand reflection-mode PPG. The common physical assumption implicit in PPG is that changes in measured light intensity are due to changes in blood volume. Light is transmitted through the anatomy of the subject, resulting in a measured intensity that depends upon the wavelength and intensity of source in addition to the optical interaction with the subject. This interaction can be expressed using the Beer-Lambert law [9], which defines the light transmittance through a medium such as blood in terms of its molar coefficient of absorption and the length of the optical path.

$$
I(\lambda)=I_{0} \exp [-\mu(\lambda) l]
$$

where $l$ is the optical path length, $\mu$ is the extinction coefficient, $I_{0}$ is the source intensity, and $I(\lambda)$ is the received intensity for a light source of wavelength $\lambda$. Whilst the physical applicability of the Beer-Lambert law is questionable [3], it has been shown that this approximation to the complex optical interactions that occur in tissue can give a good first order solution [4].

The radiative transfer equation (RTE) [43] provides a highly accurate mathematical description of the interaction of light with a physical system (Figure 1). It incorporates anisotropic scattering, which more accurately reflects the tissue characteristics; however, the increase in mathematical complexity from the use of complicated tissue models makes the application of this model extremely difficult [44, 45].

The PPG formulation of the Beer-Lambert law (eqn. 1) can be modified according to alternate approaches to the modeling of absorption, scattering and optical path length in living tissue. The models presented below are a progression from the standard Beer-Lambert Model used for PPG in pulse oximetry, allowing the inclusion of the fast growing tissue optics data [46]. By providing compatibility with increasingly popular Monte-Carlo solutions to the mathematically rigorous RTE for optical propagation in tissue, these models facilitate the discovery of relationships between the optical properties and the physiological information of the measuring site. 


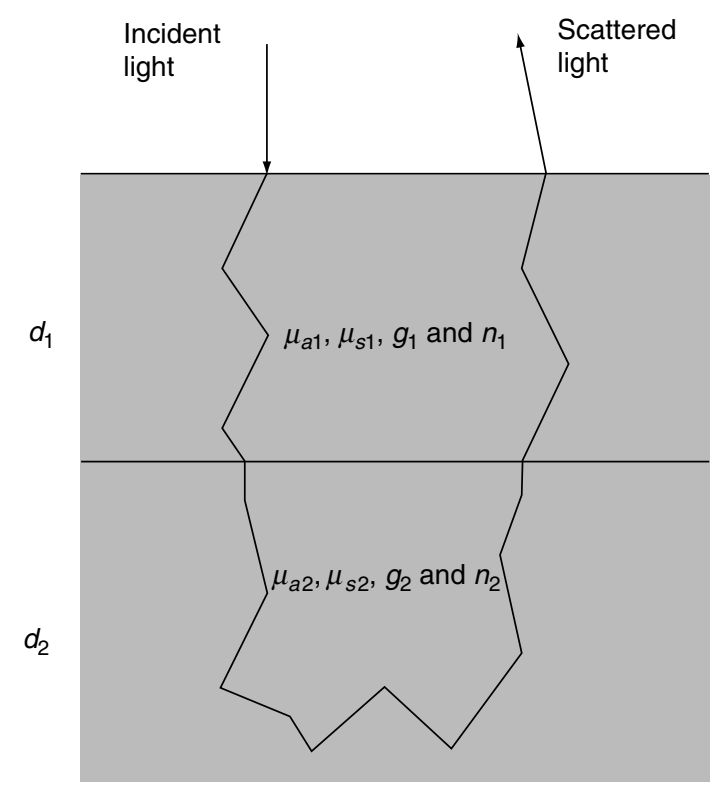

Figure 1. Diagram of a general two-layer tissue model compatible with the radiative transfer equation, showing layer thicknesses $\left(d_{n}\right)$, absorption coefficients $\left(\mu_{a}\right)$, scattering coefficients $\left(\mu_{s}\right)$, anisotropy factors $\left(g_{n}\right)$ and refractive indices $\left(n_{n}\right)$.

\subsection{Beyond the Standard Beer-Lambert Model for PPG}

Light travelling through highly scattering tissue undergoes diffusion and, on average, travels a distance proportionally greater than the source-detector separation according to the degree of scattering in the medium. Delpy et al. [47, 48] proposed an elegant and physically significant approach to the inclusion of scattering in a Beer-Lambert formulation. Assuming that the absorption is proportionally increased according to the effective path length increase due to scattering, it is possible to model the attenuation as a factor of absorption and mean optical path length:

$$
I(\lambda)=I_{0} \times \exp \left[-\mu_{a}(\lambda) L\right]
$$

where $\mu_{a}$ is the absorption coefficient, and the mean path length $L$ is a function of the reduced scattering coefficient $\mu_{s}^{\prime}$ Both of these coefficients are fundamental optical properties commonly used in tissue optics studies and are compatible with the mathematically rigorous RTE used in Monte Carlo simulations of light propagation in tissue.

The standard Beer-Lambert formulation considers the instantaneous absorption of light in a measuring site. It is well known that in a real scenario, and the 
measuring site is dynamic, thereby yielding a received intensity that varies with time. Hence formulating blood volume changes with respect to time becomes durable. Treating the measuring site as a geometrically static structure and the absorption coefficient as a dynamic optical property $\mu_{a}(t)$ with a static component $\mu_{a(\text { static }) \text {. yields: }}$

$$
\mu_{a}(\lambda, t)=\mu_{a(\text { static })}(\lambda) \times[1+\sigma \rho(\omega t)]
$$

where $\rho(\omega t)$ is the function of a normalized PPG waveform and $\sigma$ is a coefficient of pulsatility.

The dynamic component of the absorption can be attributed to the fractional change in blood volume arising from arterial blood pulsations, and the static component can be attributed to the combination of the non-pulsatile fraction of arterial blood volume and the venous blood. In this case, venous blood is treated as a static component of absorption and assuming the case where measurements are taken from a subject at rest.

The inclusion of time variance in the model can be achieved in a manner more representative of the physical system (real tissue) through the use of a time-varying mean path length:

$$
I(\lambda)=I_{0} \times \exp \left[-\mu_{a}(\lambda) L \rho(\omega t)\right]
$$

The mean path length $L \rho(\omega t)$ can be broken down into static and dynamic components, corresponding to the optical path lengths of static tissue and blood, respectively:

$$
I(\lambda)=I_{0} \times \exp \left\{-\left[\mu_{a(\text { static })}(\lambda) L_{\text {static }}+\mu_{a(\text { blood })}(\lambda) L_{\text {dynamic }} \rho(\omega t)\right]\right\}
$$

Observation of the measured intensity in both transmission and reflection modes indicates that the pulsatile component accounts for only a very small proportion of the total intensity [49]. This results from the fact that a skin vascular bed contains only a small amount of blood (2-5\%) [50], which itself experiences only a small volumetric change (around 5\%) [51] with the cardio-vascular pulse wave.

\subsection{Opto-Physiological Modeling of Transmission-Mode PPG}

An effective OP model for transmission-mode PPG on a human finger was proposed and verified through a designated protocol [52], based on the use of mean path length to account for scattering effects $[47,48]$. The model considers a set of physiologically significant layers on the measuring site and their corresponding absorption $\left(\mu_{a}\right)$, scattering (represented as detector-dependent mean path lengths $\left.L_{(\mathrm{det})}\right)$ and pulsatility coefficients $(\sigma)$, as a function of wavelength $(\lambda)$, layer $(i)$, longitudinal $(x)$ and circumferential $(\theta)$ positions: 


$$
\frac{I_{(A G)(\text { peak })}}{I_{(D C)}}(\lambda, x, \theta)=\exp \left\{\sum_{i=1}^{n}[1+\sigma(\lambda, i)] \mu_{a}(\lambda, i) L_{(\mathrm{det})}(\lambda, i)\right\}
$$

The data conversion algorithms map the distribution of ray exit points in $3 \mathrm{D}$ space $(x, y, z)$ to a cylindrical coordinate system, as would be seen on a flattened outer skin surface $(x, y)$. This is achieved by generating a central axis in $3 \mathrm{D}$ coordinates along the center of the finger model and converting the cross-sectional coordinates into equivalent angles, resulting in a surface plot of position along length in mm versus position along circumference in degrees (Figure 2). Surface analysis algorithms split these ray surfaces into square intensity buckets (virtual detectors) of arbitrary size and collect the total intensity of all rays within each bucket, thus producing an intensity distribution surface. The static and dynamic intensity surfaces are defined as

$$
I(x, y)=\sum_{x=x_{a}, y=y_{a}}^{x=x_{b}, y=y_{b}}\left[I_{t}(x, y)\right]
$$

where $I_{t}(x, y)$ is the intensity at pixel position $(x, y), I(x, y)$ is the total intensity of the bucket centered at pixel position $(x, y), x_{a}=x_{0}-d, y_{a}=y_{0}-d, x_{b}=x_{0}+d$ and $y_{b}=y_{0}+d$, where $\left(x_{0}, y_{0}\right)$ is the position on the flattened finger surface, and $d$ is the square bucket half-size. Eqn. 7 is used to derive the ratio of ratios $R$ as a function of surface position:
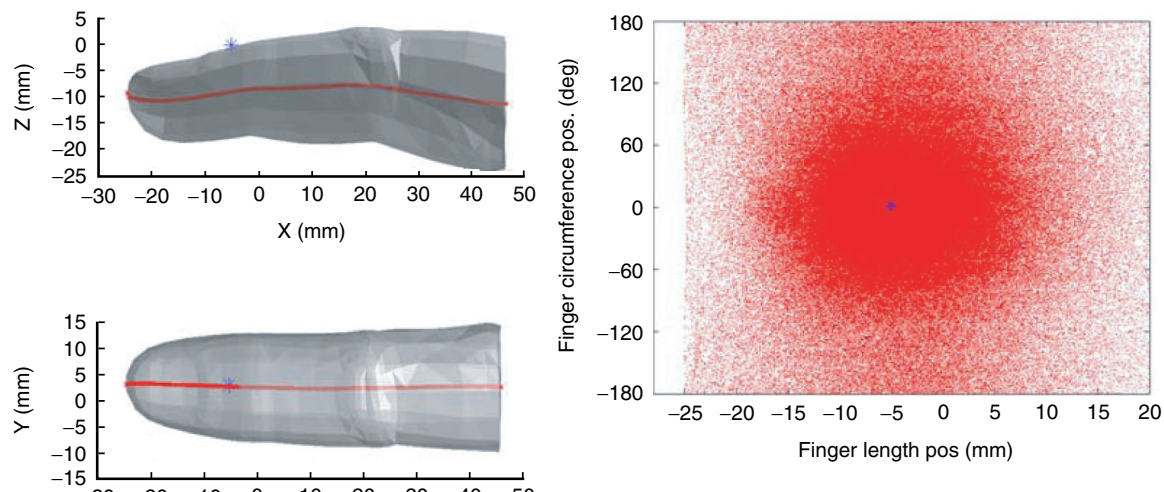

Figure 2. Outer skin model with central finger axis highlighted (left), and resultant plot after conversion of transmitted ray coordinates from $3 \mathrm{D}$ to cylindrical (2D) coordinates (right). The light source position is marked by an asterisk $(*)$. 


$$
R(x, y)=\frac{\left[I_{R E D(A C)(d i a)}(x, y)-I_{R E D(A C)(s y s)}(x, y)\right] / I_{R E D(D C)}(x, y)}{\left[I_{I R(A C)(\text { dia })}(x, y)-I_{I R(A C)(\text { sys })}(x, y)\right] / I_{I R(D C)}(x, y)}
$$

\subsection{Opto-Physiological Modeling for Reflection-Mode PPG}

To model reflection-mode PPG, the layered absorption $\mu_{a}$ as a function of wavelength $\lambda$ and layer $k$, and the detector-dependent pulsatile mean path length $L_{(A C)}$ as a function of layer $k$ and time $t$ are considered for an arbitrary detector position, where the dynamic component $I_{(A C)}(k)$, representing specific layer blood perfusion can be derived as:

$$
I_{(A C)}(k, t)=I_{(A C)} \times \frac{\mu_{a(A C)}(k, \lambda) \times L_{(A C)}(k, t)}{\sum_{i=1}^{n} \mu_{a(A C)}(i, \lambda) \times L_{(A C)}(i, t)}
$$

where $I_{(A C)}$ is the pulsatile component of the imaging PPG signal acquired from the arbitrary detector position. By considering all detector positions over the designated tissue, 3-D mapping of segment blood perfusion can be achieved. Consequently, the static blood perfusion component of a specific layer $k$ can be derived with its weight $w_{(D C)}(k)$ and the total static component as:

$$
\begin{aligned}
I_{(D C)}(K) & =I_{(D C)} \times w_{(D C)}(k)=I_{(D C)} \times \frac{\exp \left[-\mu_{a(D C)}(k, \lambda) \times L_{(D C)}(k)\right]}{\prod_{i} \exp \left[-\mu_{a(D C)}(i, \lambda) \times L_{(D C)}(i)\right]} \\
& =\frac{I_{(D C)}}{\prod_{i^{\prime} \neq i}^{k} \exp \left[-\mu_{a(D C)}\left(i^{\prime}, \lambda\right) \times L_{(D C)}\left(i^{\prime}\right)\right]}
\end{aligned}
$$

where $\mu_{a}(D C)$ is the wavelength-dependent absorption coefficient of static component of tissue in $\mathrm{mm}^{-1}$, and $L_{(D C)}$ is the static component of mean path length (MPL) in mm. Similarly, the perfusion $I_{(A C)}$ of a specific layer $k$ can be expressed as the product of the total perfusion and $w_{(A C)}(k)$ :

$$
\begin{aligned}
I_{(A C)}(k) & =I_{(A C)} \times w_{(A C)}(k)=I_{(A C)} \times \frac{\mu_{a(A C)}(k, \lambda) \times L_{(A C)}(k)}{\sum_{i} \mu_{a(A C)}(i, \lambda) \times L_{(A C)}(i)} \\
& =\frac{I_{(A C)} \times\left(L_{(A C)}(k)\right.}{\sum_{i} L_{(A C)}(i)}
\end{aligned}
$$

where $\mu_{a(A C)}$ is the wavelength-dependent absorption coefficient of arterial blood in $\mathrm{mm}^{-1}$, and, $L_{(A C)}$ is the dynamic component of mean path length in $\mathrm{mm}$. 


\subsection{Monte Carlo Simulation}

A Monte Carlo (MC) simulation platform was developed for this work for the purpose of providing a detailed description of the light propagation in a multi-layered tissue with dynamic blood volume changes. The platform utilizes two commercially available software packages. OptiCAD (OptiCAD Co., USA) comprises an MC ray-tracing engine where the coordinates of traced ray vertices is generated, thereby making the calculation of mean path length a straightforward process. MATLAB (Mathworks Co., USA) was chosen as a development language for data processing algorithms, and for preparation of the 3D models used in simulation. Post data processing was performed in MATLAB through the use of custom algorithms specific to the MC ray trace outputs.

In the mathematical tissue models presented in the previous section and in the corresponding virtual models used in the simulation, the individual layers are assumed to be homogeneous, and each layer is characterized by a bulk absorption coefficient $\mu_{\mathrm{a}}$, scattering coefficient $\mu_{s}$, anisotropy factor $g$ and refractive index $n$. Contrary to this assumption, the layers that make up tissue are neither homogeneous nor well-defined and planar. Nonetheless, results of a previous study [53] indicate that the macroscopic optical properties of whole tissue samples and tissue homogenates are similar.

The anisotropy factor $g$ was set to 0.8 [44]. Although certain components of the tissue volume such as collagen [54] and red blood cells [55] are more forwardscattering, the value of 0.8 is more representative of overall dermal scattering. The refractive index for all the layers was set to be 1.4 based upon the results of a relevant study [56]. It should be noted that the model layer interfaces are artificial; the actual structural interfaces in tissue (e.g., vessel walls) are at a finer level of detail than the model represents. The simplifying assumptions made here are similar to those made in other studies [41, 44, 57].

In blood perfused layers, the absorption coefficients of dermis and other highly vascularised tissues in the near infrared spectral region depend on the blood content. Also, the scattering in the dermis and in the sub-dermal tissues dominates absorption [58]. The structures that contribute most to scattering in skin are collagen fibre bundles and red blood cells, which cause highly forward-directed scattering [59]. The wavelengthdependent optical properties of blood and tissue from literature [38, 39, 45, 57] are listed in Table 1.

The total blood fraction (nominal 5\%) is the mean concentration of blood in the total tissue volume during the diastolic state. Layer thickness $d(k)$, and blood fraction $f(k)$ for each layer in the diastolic state with nominal total blood fraction of 5\% [51] is listed in Table 2.

The absorption and scattering coefficients of the virtual skin tissue model were computed as the combination of the coefficients of whole blood and bloodless tissue weighted by their respective volume fractions:

$$
\mu=\left(1-f_{\text {blood }}\right) \times \mu_{\text {bloodless }}+f_{\text {blood }} \times \mu_{\text {blood }}
$$

where $f_{\text {blood }}$ is the total blood volume fraction, $\mu_{\text {bloodless }}$ is the absorption or scattering coefficient of bloodless tissue in $\mathrm{mm}^{-1}$, and $\mu_{\text {blood }}$ is the absorption or scattering coefficient of whole blood in $\mathrm{mm}^{-1}$. 
Table 1. Optical properties of blood and bloodless tissue at $650 \mathrm{~nm}$ and $870 \mathrm{~nm}$ illumination

\begin{tabular}{llll}
\hline Var. & \multicolumn{1}{c}{ Meaning } & $\mathbf{6 5 0} \mathbf{n m}$ & $\mathbf{8 7 0} \mathbf{~ n m}$ \\
\hline$\mu_{a, \text { bloodless }}\left(\mathrm{mm}^{-1}\right)$ & absorption, bloodless tissue & 0.0285 & 0.0245 \\
$\mu_{a \text {,blood }}\left(\mathrm{mm}^{-1}\right)$ & absorption, blood & 0.397 & 0.583 \\
$\mu_{s, \text { bloodless }}\left(\mathrm{mm}^{-1}\right)$ & scattering, bloodless tissue & 7.150 & 4.450 \\
$\mu_{s, \text { blood }}\left(\mathrm{mm}^{-1}\right)$ & scattering, blood & 161.374 & 106.888
\end{tabular}

Table 2. Layer thickness $d(k)$ and blood fraction $f(k)$ for each layer in the non-pulsatile state

\begin{tabular}{llll}
\hline $\mathbf{k}$ & \multicolumn{1}{c}{ Layer name } & $\boldsymbol{d}(\mathbf{m m})$ & $\boldsymbol{f}(\boldsymbol{\%})$ \\
\hline 1 & Epidermis & 0.06 & 0 \\
2 & Dermis & 0.2 & 0 \\
3 & Dermis Plexus Superficialis & 0.2 & 5.56 \\
4 & Dermis & 0.8 & 4.17 \\
5 & Dermis Plexus Profundus & 0.6 & 20.4 \\
6 & Hypodermis & 8.0 & 4.17
\end{tabular}

Since each layer was assumed to be of uniform thickness, its volume was expressed by its thicknesses alone. The pulsatile fraction is the fraction of the total tissue volume displaced by the arterial pulse. A nominal diastolic blood fraction $f_{\text {blood }}$ was $5 \%$ with nominal pulsatile fraction $f_{\text {pulse }}$ taken as $0.25 \%$ [51]. The pulsatile blood fraction was applied uniformly to all perfused layers:

$$
f_{\text {pulse }}(k)=\left[\frac{d(k)}{d}\right] \times f_{\text {pulse }}
$$

Table 3. Optical properties of each tissue layer for $870 \mathrm{~nm}$ illumination source

\begin{tabular}{lllll}
\hline Layer $(\boldsymbol{k})$ & $\begin{array}{c}\mu_{\boldsymbol{a} \text {,non-pulsatile }} \\
\left(\mathbf{m m}^{-\mathbf{1}}\right)\end{array}$ & $\begin{array}{c}\mu_{\boldsymbol{s} \text {,non-pulsatile }} \\
\left(\mathbf{m m}^{\mathbf{- 1}}\right)\end{array}$ & $\begin{array}{c}\mu_{a, \text { pulsatile }} \\
\left(\mathbf{m m}^{-\mathbf{1}}\right)\end{array}$ & $\begin{array}{c}\mu_{\text {s,pulsatile }} \\
\left(\mu^{-\mathbf{1}}\right)\end{array}$ \\
\hline Epidermis (1) & 0.02450 & 4.4500 & 0.02450 & 4.4500 \\
Dermis (2) & 0.02450 & 4.4500 & 0.02450 & 4.4500 \\
Dermis Plexus superficialis (3) & 0.05556 & 10.1455 & 0.05559 & 10.1507 \\
Dermis (4) & 0.04779 & 8.7217 & 0.04791 & 8.7424 \\
Dermis Plexus Profundus (5) & 0.1383 & 25.3166 & 0.13840 & 25.3321 \\
Hypodermis (6) & 0.04779 & 8.7217 & 0.04892 & 8.9294
\end{tabular}


where $f_{\text {pluse }}$ is the overall pulse blood fraction of the tissue, $f_{\text {pluse }}(k)$ is the pulse blood fraction of layer $k, d(k)$ is the thickness of layer $k$ in $\mathrm{mm}$, and $d$ is the total thickness in $\mathrm{mm}$.

In considering tissue with dynamic blood volume changes, the arterial pulsation was simulated by adding the pulsatile blood into the non-pulsatile tissue and thus changing the volume fraction of each component in tissue. The scattering and absorption properties of tissue are changed in the non-pulsatile and pulsatile stages by linear combination of the coefficients of each component weighted by its respective fraction. Consequently, values for detector-specific mean path lengths, $L_{(\mathrm{det})(\mathrm{static})}$ and $L_{\text {(det)(dynamic), }}$ were calculated based upon the tissue models with pulsatile and nonpulsatile optical properties, respectively.

\section{EMPIRICAL STUDIES}

The experiments of transmission-mode and reflection-mode implementations of PPG were carried out to investigate transmission- and reflection-mode OP models. These experiments represent the first steps towards the validation of opto-physiological models developed and simulated in section 2.

The processing pipeline for a standard PPG system can be divided into two stages, namely the main and post-processing stages (Figure 3, left). The pipeline of a typical iPPG system can be said to comprise an additional pre-processing stage, involving the extraction of an array of meaningful PPG signals from the images captured by the system (Figure 3, right).

\subsection{Effective Signal and Image Processing Procedures}

The pre-processing stage of an iPPG system involves the extraction of one or more PPG signals from a sequence of images. To extract a signal from raw iPPG data, the average value of pixels within an arbitrary region of interest (ROI) is taken from each frame. Pre-processing also comprises an optional image stabilization process that is performed prior to ROI signal extraction.

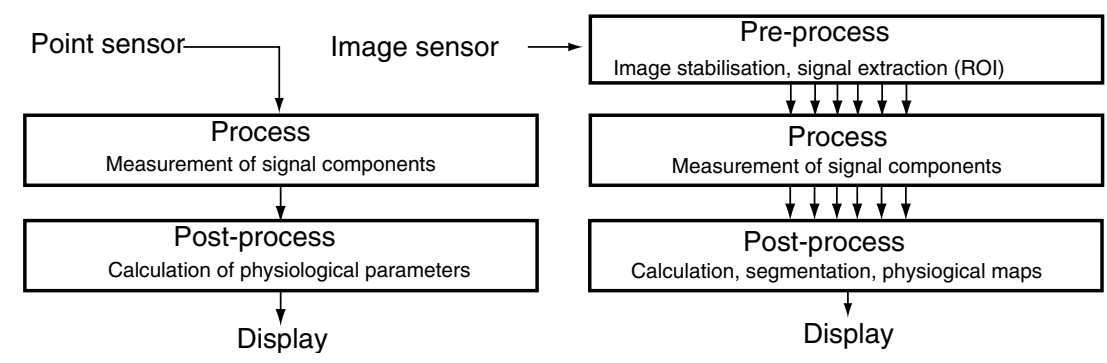

Figure 3. Processing pipelines for point- and imaging-measurement PPG systems. 
The main processing stage involves the measurement of static (tissue), quasi-static (breathing) and dynamic (heart) components of PPG signals. For a point measurement system, i.e., pulse oximetry probe, the number of signals passed to the main process is typically a function of the number of illumination wavelengths, whereas for an iPPG system, the number of signals is typically the product of the number of wavelengths and ROI. The main processing stage employs time (e.g., filtering), frequency (e.g., spectral peak detection), or joint time-frequency domain (e.g., Time-Frequency Representation) techniques to measure PPG signal components.

- $\quad$ Filtering is used to separate the tissue, breathing and cardiac components of the signal according to their frequency bands, where the DC amplitude is the tissue component, the breathing component is typically less than $0.5 \mathrm{~Hz}$, and the heart (AC) component typically spans the $1-5 \mathrm{~Hz}$ range $[60,61]$. Filtering is performed on all raw PPG signals in this study.

- $\quad$ Once separated, the amplitudes of these components are readily accessible, and the instantaneous frequencies of the non-static (breathing and heart) components can be attained by measuring the time elapsed between adjacent peaks or troughs. The peak-to-peak intensity of the AC components from filtered PPG signals can be determined by taking the difference between the amplitudes of adjacent peaks and troughs in the AC component.

- Reliant on the Fast Fourier Transform (FFT) algorithm, the conversion of raw signal data into the frequency domain allows the measurement of amplitudes and frequencies of PPG signal components via straightforward identification of peaks in the resultant frequency spectrum. The fidelity of peaks in its frequency spectrum is highly sensitive to both the sample rate of the signal and the length of the signal segment (number of samples) used in the FFT, thus posing an inherent tradeoff between temporal and spectral characterization of the PPG data. An increased characterization of lower frequencies requires a greater number of samples in the FFT, and since the vast majority of energy in a PPG signal is located close to DC, the temporal/spectral tradeoff is aggravated.

The post-processing stage comprises the calculation of application-specific physiological parameters using temporal and/or spectral data, and consists of operations such as statistical calculations and unit conversions (scaling and offsetting) of the measurements acquired in the previous stage. Physiological parameters that are commonly calculated include heart rate (HR), respiration rate (RR) and pulse oximetry $\left(\mathrm{SO}_{2}\right)$. HR and $\mathrm{RR}$ are determined by taking the acquired frequency information (in $\mathrm{Hz}$ ) and resolving to corresponding values of beats and breaths per minute, respectively. The determination of $\mathrm{HR}$ involves taking the time elapsed of the samples between two adjacent peaks in the AC component of the PPG signal, dividing by the sampling rate of the signal (samples/second), and multiplying by 60 to give the instantaneous heart 
rate in beats per minute. Pulse oximetry requires PPG signals that are simultaneously acquired under at least two distinct wavelengths of illumination and involves calculating the ratio of the amplitudes of the cardiac components to the corresponding tissue components (also a relative measure of perfusion), calculating the ratio of these normalized cardiac components (ratio of ratios) and acquiring the $\mathrm{SO}_{2}$ value corresponding to that ratio from a calibration curve. In an iPPG system, the physiological parameters calculated in this process are spatially defined in the 2-D plane corresponding to the tissue surface being imaged. At this stage, it is therefore common to generate a 2-D map of the physiological parameters. Furthermore, a multilayered tissue model can be applied at this stage, yielding a 3-D map of the layered dynamic blood perfusion.

In an offline system, PPG information is readily available and can be processed with no performance restrictions. For point measurement techniques, the dynamic (AC) component of the signal is generally obtained by band-pass filtering, and the static (DC) component of the static volume is obtained through low-pass filtering, e.g., $10^{\text {th }}$ order Butterworth [60]; the $\mathrm{AC}$ component corresponds to $I(\lambda)$ as per eqn. 5, which is obtained by applying a band-pass filter on the signal. In the case of imaging, ROI can be arbitrarily chosen to investigate spatial variations in blood perfusion. In the experiments detailed in this section, the common procedure used for the processing of PPG signals involves the removal of the DC component from the captured PPG waveform, leaving a typical PPG AC pulsatile signal which contains both HR and RR components. These components can be measured in the time domain via band-pass separation, or in the frequency domain via identification of peaks corresponding to each component.

\subsection{Validation of a Monte Carlo Platform for Opto-Physiological Modeling}

An accurate virtual 3D anatomical model (Zygote Media Group, USA) of a male adult finger was used to numerically solve the RTE for the case of a point source LED light at $633 \mathrm{~nm}$ and $850 \mathrm{~nm}$ [63], placed near the standard position found in commercial probes. The simulation results were processed using eqn. 6 to distinguish localized light intensities that vary due to the cardiovascular cycle from those that are static, thus providing a means to construct static and dynamic intensity distributions mapped to the surface of the finger. Sensor responses were generated by scanning a square bucket of arbitrary size in $0.1 \mathrm{~mm}$ increment from -1 to $1 \mathrm{~mm}$ longitudinally and from 0 to $360^{\circ}$ circumferentially.

For validation of Monte Carlo simulation results, a CMOS camera (Mightex, USA) was mounted on a rotating platform that allowed the camera to maintain a constant distance and alignment with respect to the subject's finger (Figure 4) [52]. Sets of frames were captured at each position between $90^{\circ}$ and $270^{\circ}$ in $9^{\circ}$ increment (a total of 21 positions), where $180^{\circ}$ is the standard transmittance mode sensor position with respect to the light source. For each position, two sets of 200 frames were captured at $40 \mathrm{fps}$ in immediate succession, i.e., for both red and infrared illumination. For each of the sets, the mean peak-to-peak intensity and mean intensity was determined from windows of size $10 \times 10$ pixels $\left(1\right.$ pixel $\left.=0.156 \mathrm{~mm}^{2}\right)$, resulting in two additional 


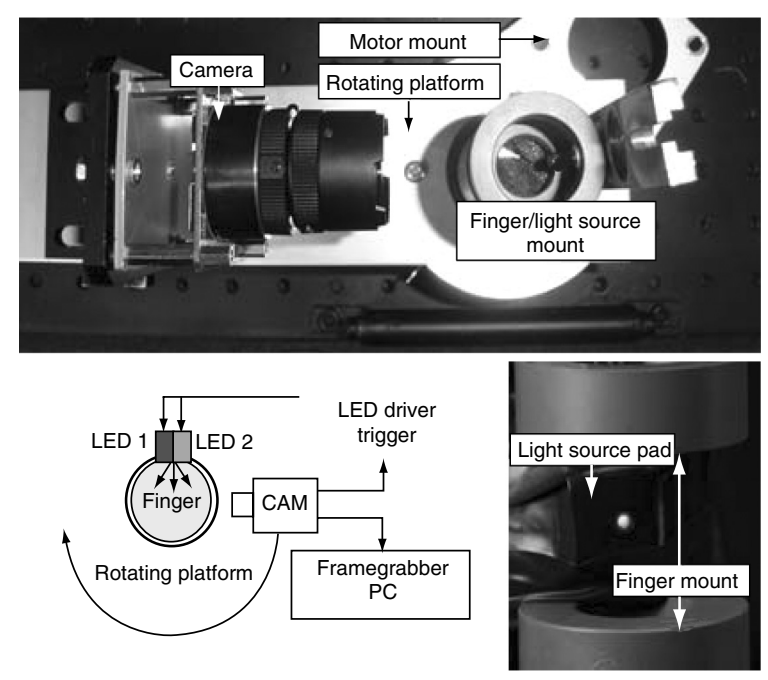

Figure 4. Validation platform for Monte Carlo virtual environment. The rotating platform (top) was powered by a stepper motor, programmed to rotate the camera in $9^{\circ}$ steps. The dual-wavelength LED light source was housed in an ergonomic pad within the finger mount into which subjects inserted their index finger (bottom-right) [52].

frames representing the AC and DC intensity distributions of that set. Finally, the AC and DC frames were trimmed and concatenated to form continuous intensity distributions from $90^{\circ}$ to $270^{\circ}$. The Loughborough University Ethics Committee approved the protocol for this empirical study.

Figure 5 shows that the ratio of ratios in the range $120^{\circ}-230^{\circ}$ is stable with a variation of \pm 0.1 , showing peaks corresponding to positions of blood vessels. The drops at positions closest to the light source reflect the loss of dynamic signals due to saturation of the red channel. A positive correlation can be seen between corresponding signals at different wavelengths, and a negative correlation can be seen between QuasiDC and AC components for positions closest to the light source, i.e., $90^{\circ}$ and $270^{\circ}$.

A study was performed on this validation platform [52], in which the fingers of 10 subjects were mapped and the resultant distributions were used to construct ratio of ratios distributions. The protocol was approved by the Loughborough University ethics committee. The statistical analysis shown in Figure 6, indicates an even distribution of dynamic signal strength in the range of $120^{\circ}-240^{\circ}$ and suggests a tendency for stronger signals in the range of $180^{\circ} \pm 40^{\circ}$.

\subsection{Perfusion Mapping using Imaging Photoplethysmography}

For the iPPG system shown in Figure 7, the camera (Mikrotron GmbH MC1311) has a maximum resolution of $1280 \times 1024$ pixels and uses a $1.25^{\prime \prime C M O S}$ sensor with square 


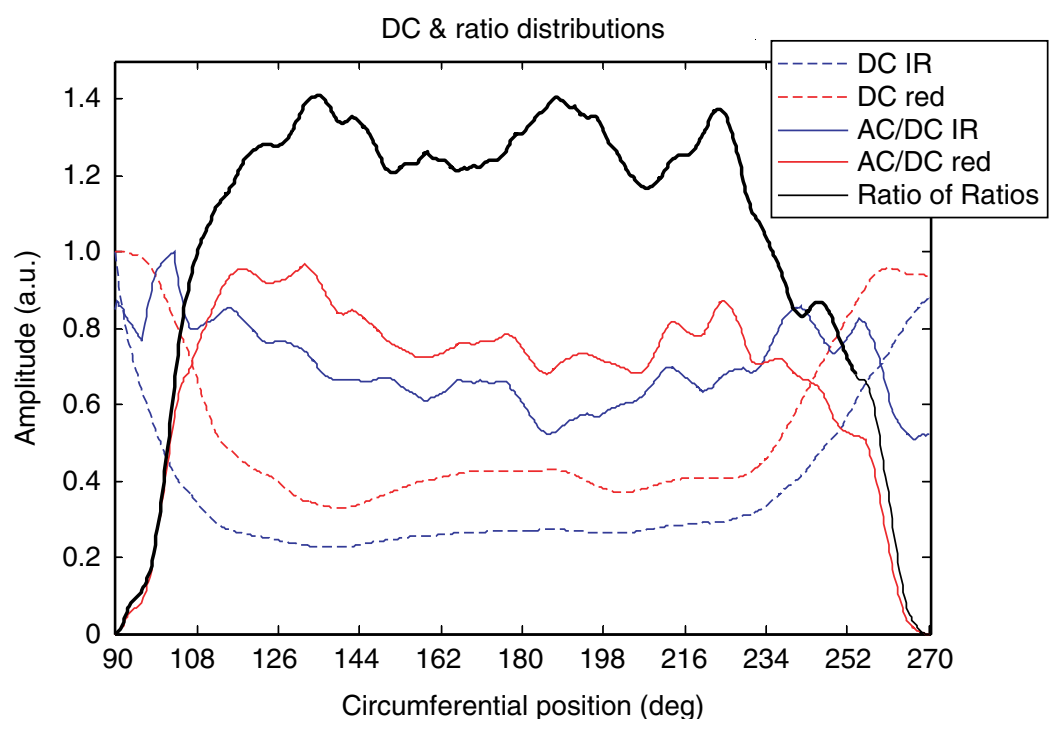

Figure 5. $\quad \mathrm{DC}$ and $\mathrm{DC}$ normalized peak-to-peak AC distributions from 12 bit camera data sweep with window of size $6 \times 6$ pixels, and light source at 0 deg [52]. From each signal, the Quasi-DC component (dotted curve) was extracted by low-pass filtering, and the AC component was extracted by taking the difference between detected peak and trough envelopes. Quasi-DC and AC components were then used to generate corresponding $\mathrm{AC} / \mathrm{DC}$ and ratio of ratios components (solid curves). Each point of the above distributions corresponds to the mean of the corresponding signal component.

pixels measuring $12 \mu \mathrm{m}$. The pixels are encoded using 10 bits, making the imaging sensor capable of detecting the weak arterial pulsation. A custom built dual-wavelength LED ring light source $(\lambda 1: 650 \mathrm{~nm}, \lambda 2: 870 \mathrm{~nm})$ with a parabolic reflector (DIA: $18 \mathrm{~cm}$, O.L: $6.5 \mathrm{~cm}, \mathrm{~B} \& \mathrm{Q}, \mathrm{UK})$ was mounted around the camera zoom lens (Computar, Japan) to provide a collimated and uniform illumination on a plane perpendicular to the axis of the lens [64]. The ring light consisted of 20 LEDs: 10 with a peak wavelength of $650 \mathrm{~nm}$ (TRC650SMD0603, WelTek Co. Ltd., Taiwan), each emitting $1.0 \mathrm{~mW}$ power at a forward current of $20 \mathrm{~mA}$, and another 10 with a peak wavelength of $870 \mathrm{~nm}$ (TRC870SMD0603, WelTek Co. Ltd., Taiwan) emitting $1.3 \mathrm{~mW}$ power individually. The arrangement of the LEDs is shown in the right inset of Figure 7. A control circuit with a microcontroller (PIC16F876A, MicroChip Inc., USA) alternately powered each wavelength group of LEDs such that the light output duration for each wavelength was constant. The relative illumination timings are illustrated in the left insets of Figure 7, where the camera was triggered every time there was a switch of wavelength. The frame rate was set at $30 \mathrm{fps}$ (15 fps for each wavelength), which was sufficient to recover the shape of the PPG arterial 

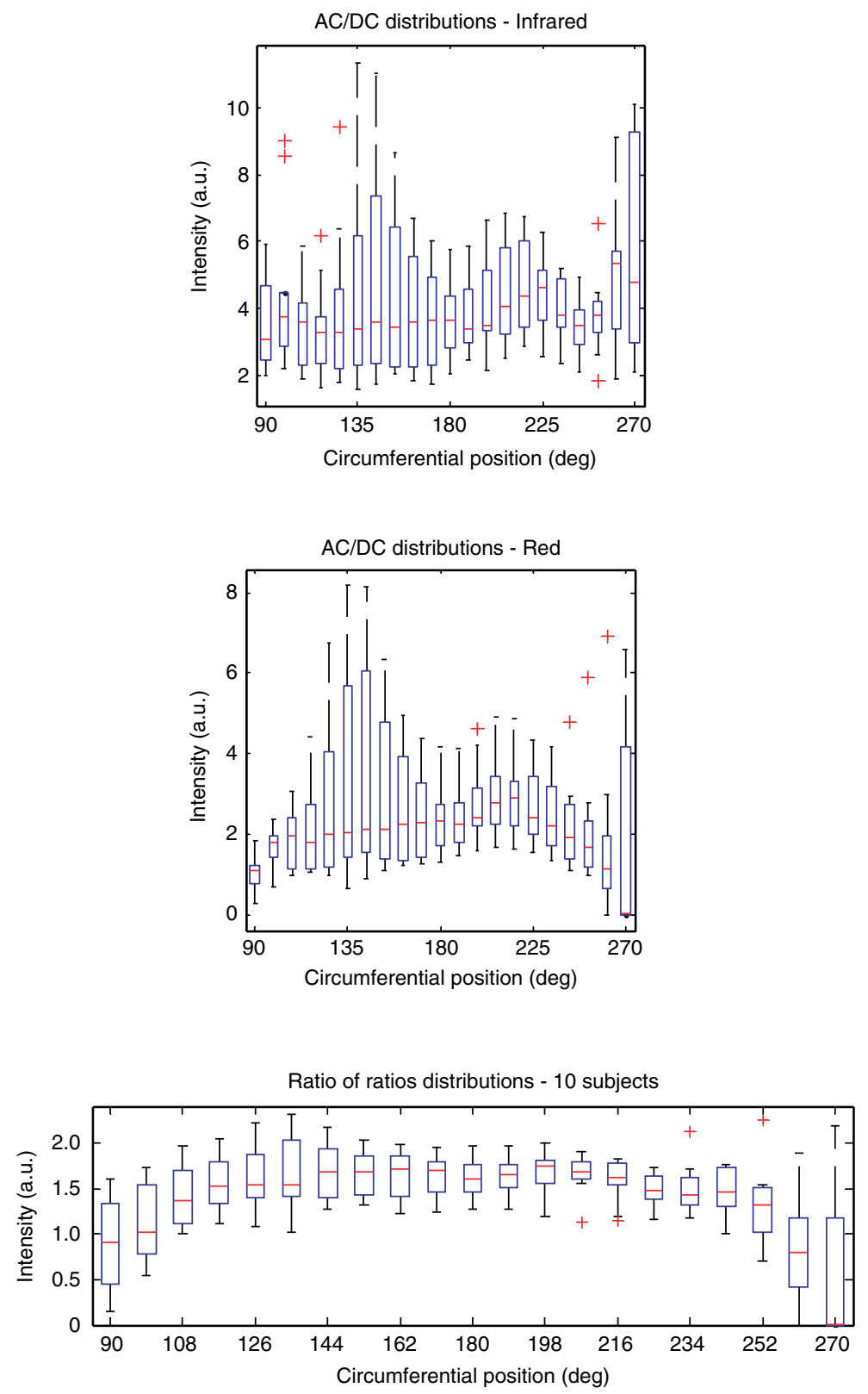

Figure 6. Box \& whisker plot of normalized AC distributions of IR (left) and red channels (right), and ratio of ratios distributions (bottom) for a 10 subject study [52]. 


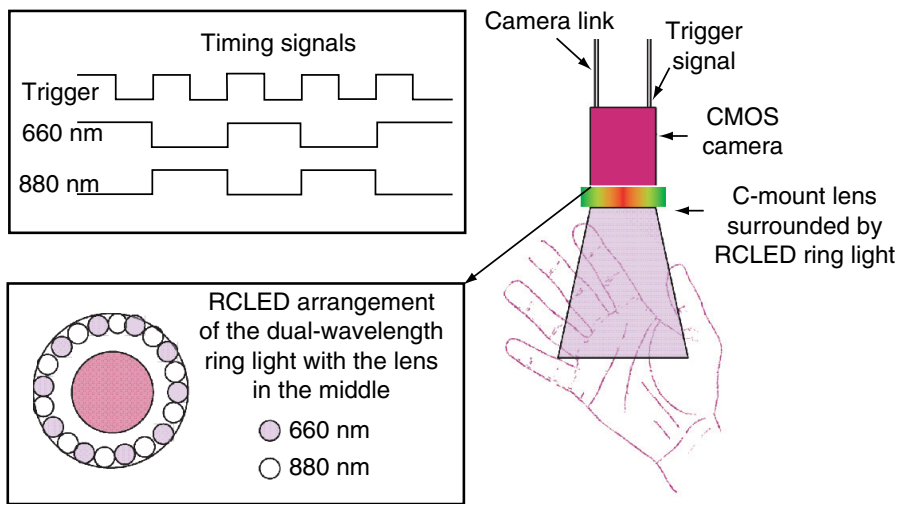

Figure 7. Illustration of iPPG system. The depicting illumination and imaging geometry (right); the relative timing signals (top); and the LED arrangement of the dual-wavelength ring light (bottom) [64]. Reproduced with permission.

waveforms. We observed arbitrary areas of the skin surface, from a few square millimeters to several square centimeters. The camera was mounted on an optical bench with the lens positioned approximately $13 \mathrm{~cm}$ away from the palm of the subject (Figure 5). The protocol was approved by the Loughborough University ethnics committee.

The spatial resolution of the 2-D and layered perfusion map is determined by the size of the ROI, which is affected by the resolution of the captured frame, the quality of the iPPG signal and the tissue's spatial point spread function. The optical resolution for the C-mount zoom lens with the CMOS camera was calculated as $1.0 \times 1.0 \mu \mathrm{m}^{2}$ of $650 \mathrm{~nm}$ illumination and $1.5 \times 1.5 \mu \mathrm{m}^{2}$ of $870 \mathrm{~nm}$ which are much smaller than the pixel size $12 \times 12 \mu \mathrm{m}^{2}$ of the CMOS camera. Given the uniform illumination and the homogenous tissue model, the mean path length $L_{(A C)}$ for all layers remains constant as long as ROI size is above the optical spatial resolution of the deepest layer, $15.32 \mathrm{~mm}$, corresponding to the size of ROI $100 \times 100$ pixels $^{2}$. Thus the resolution of the captured frame is mainly determined by the pixel size of the camera.

By employing the principles relating to PPG, blood perfusion maps were generated from the power of the PPG signals in the frequency domain. The acquired image frames were processed as follows:

(1) Ten seconds worth of image data were recorded with the acquisition system.

(2) The average fundamental frequency of the PPG signal was manually extracted $(1.4 \mathrm{~Hz})$.

(3) The pixel values of all images were extracted for a particular pixel position to form a pixel value vector in the time domain.

(4) The 64-point Fast Fourier Transform (FFT) was calculated for the signal at the pixel position. 
(5) The Power of the FFT tap corresponding to the PPG fundamental frequency was copied into the same pixel coordinates of a map of overall blood perfusion.

Repeating procedures (3) to (5) for all the remaining pixels (clusters) provides a new matrix (image) whose elements (pixels) depend on the power of the detected blood volume variation. This technique allows the generation of a blood perfusion map, as a high PPG power can be attributed to high blood volume variation and ultimately to blood perfusion.

PPG information such as heart rate is clearly observed from one wavelength in Figure 8 (left). The time-frequency representation of the pulsatile components derived from iPPG signals is presented for a time window of 10 seconds (150 frames) in Figure 8 (right). The fundamental frequency (i.e., HR frequency) and $1^{\text {st }}$ harmonic can be identified in Figure 8 (right), indicating that both the HR and the shape of the plethysmogram were determined.

The iPPG signal can be quantitatively related to the optical properties of each layer. The conversion of a 2-D map to a 3-D perfusion map is performed by applying the optophysiological model at each ROI on the 2-D map, where the model allows for the determination of the relative contributions of different layers to the ROI-dependent perfusion of the 2-D surface map. By mapping the layered components of each ROI, it is possible to illustrate the relationship between segmented AC and DC signals, and the relationship between segmented and whole iPPG signals. Figure 9 shows the segmented perfusion from a hand under $870 \mathrm{~nm}$ illumination, normalized with respect to the DC intensities, and plotted in a 3-D format to illustrate the layered dynamic blood perfusion.

The iPPG signal is quantitatively related to the optical properties of each layer. The layered perfusion mapping illustrates the relationship between segmented AC and DC signals, and the relationship between segmented signal and the total iPPG signal.
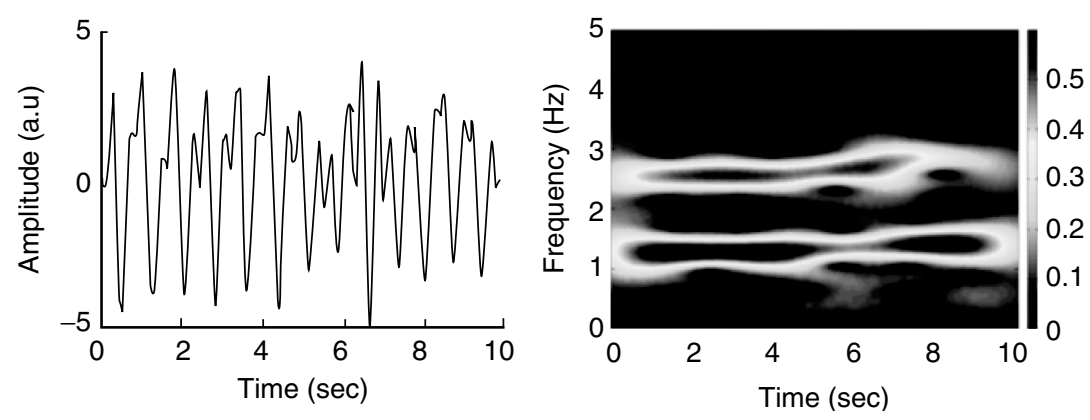

Figure 8. AC (filtered) component from $870 \mathrm{~nm}$ source (left) and the corresponding joint time-frequency diagram of the AC component of $10 \mathrm{sec}$ duration, computed using pseudo Wigner-Ville distribution (right). 


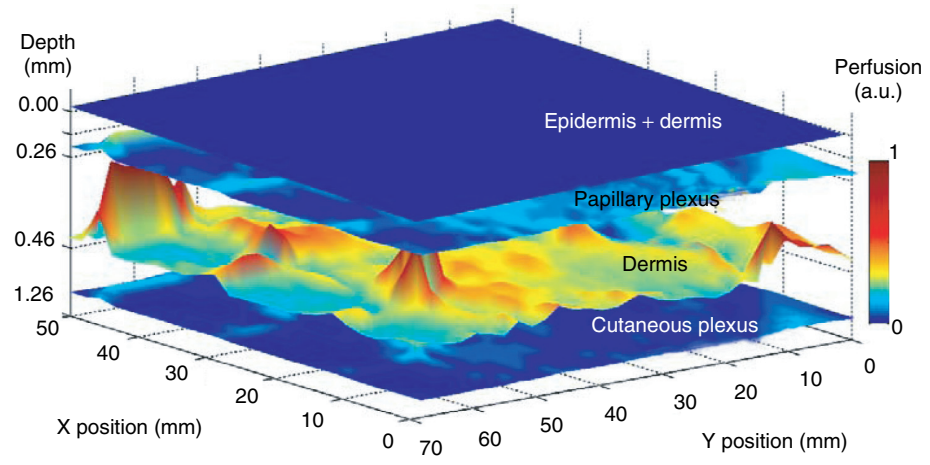

Figure 9. Segmented perfusion mapping of the multi-layer skin tissue of palm under $870 \mathrm{~nm}$ illumination, generated using eqns. 9 and 10 on the PPG signals derived from each pixel position of the images acquired from the iPPG setup [64]. The $y$ dimension runs from the base of the palm to the base of the fingers; the $x$ dimension runs across the palm from left to right; the depth coordinate of the top of each layer is shown.

\section{DISCUSSION}

High correlation is apparent between corresponding distributions at different wavelengths, thereby suggesting that the two light sources are trans-illuminating the same body of tissue at each circumferential position. A smoother response from red illumination is apparent, as would be expected from a higher degree of optical diffusion. This is substantiated by the fact that the scattering coefficients of all relevant human tissue types under red illumination are notably higher than those corresponding to IR. Ratio of ratios distribution shows a high variability of \pm 0.3 in the central positions. Also, an offset is visible between both static and dynamic responses with respect to illumination wavelength. This is evidence of variations in light source and finger positions due to individual recording sessions for each wavelength.

In order to obtain quality PPG signals, the uniform illumination on the skin surface of the subject seemed vital to capture pulsatile waveforms from the ROI. Hence the distance between the cameras coupled the illumination sources $(650 \mathrm{~nm}$ and $870 \mathrm{~nm})$ and the subject was maintained at 20-30 cm away. The perfusion map in Figure 9 is normalized with respect to DC intensities, thereby providing a visual representation of signal quality with respect to position on the skin surface. As the perfusion map is layered, it also illustrates the relationship between segmented signals and the total iPPG signals. The spatial resolution of the 2-D and layered perfusion mapping is determined by the size of ROI, which is affected by the resolution of the captured frame, the quality of the iPPG signal and the tissue's spatial point spread function. Through the perfusion mapping, the iPPG signal is quantitatively related to the segment optical properties of each layer. By mapping the layered components of AC/DC ratio of each ROI, all the points within layered tissue's spatial point spread function in Figure 9 contribute to the ROI-dependent AC/DC ratio. The perfusion 
map indicates that the deep dermis layers are the main contributor to blood perfusion up to $80 \%$ of the total perfusion, a result in accordance with simulation setup and empirical value. As the top two layers contain no pulsatile blood, there is no blood perfusion. The deeper dermal structures contain the arterioles, venules, and shunting vessels to feed and drain the capillary network and aim at maintaining an adequate body temperature [68]. As the top two layers contain no pulsatile blood, there is a negligible blood perfusion. Based upon the current model and simulation settings, the geometry of individual layers of tissue is static and identical, and the normalized layered power mapping can mainly provide information about the dynamic optical properties of tissue. Main issues limiting the accuracy of the current approach are the motion artefact and the uncertainty of the degree of correspondence between this model and the real tissue.

There is an expected variation between the model predictions and the in vivo experimental outputs due to the difficulty to quantify surface reflection and the sensitivity of the current approach based upon a predefined tissue structure. As the parameters of the predefined tissue approach real life, an increasing correlation is expected between the outputs of the opto-physiological model and empirical measurements. This research would greatly benefit from a close-to-real tissue structure with accurate measurement of the internal makeup of the tissue bed.

In all the above experiments, acquisition of real data, generation of simulated data, and post-analysis of data were performed in separate stages. The volume of data produced, particularly from the image acquisition and simulation platforms, poses a significant challenge when working towards a real-time system. Some work has been done towards developing a scalable system that acquires images, processes the image data, and outputs physiological information in real-time [69].

In a clinical setting, physiological assessment relies on the real-time execution of signal processing codes as a key to enabling safe, accurate and timely decision-making, allowing clinicians to make important decisions and to perform medical interventions based on hard facts that are derived in real-time from physiological data [65, 66]. This is an area where advanced computer architecture concepts, routinely utilized in highperformance consumer and telecoms Systems-on-Chip (SoC) [67], can potentially provide the required data streaming and execution bandwidth to allow for the real-time use of the opto-physiological models presented in this paper. These models would otherwise be used off-line (in batch mode) using more established techniques and platforms (e.g., sequential execution on a PC host).

\section{CONCLUSION}

On-going progress is being made in a multitude of areas concerning photoplethysmographic cardiovascular assessment. Significant paradigm shifts in photoplethysmographic measurement techniques can be categorized into two themes, namely the migration from point (1D) to image (2D) sensing, and the migration from contact to non-contact sensing. This paper reviews a body of research comprising two OP models that have led to significant progress in the design of point and imaging sensors for the acquisition and interpretation of cardiovascular performance. 
The Monte Carlo validation approach to OP modeling relies on a process of finetuning the accuracy of characterization for both simulation and empirical validation. The approach demonstrates its functionality at a qualitative level and provides a path towards increasingly comprehensive descriptions of the mechanisms involved not only in point measurement, i.e. pulse oximetry, but also in the layered perfusion mapping, i.e. iPPG. In the present OP models, the changes of $\mu_{a}$ and $\mu_{s}$ can be related to the dynamic change, and this means the dynamic part of the OP model can be validated through in vitro phantom validation. The precision validation of the dynamic component can also be achieved by using a phantom with an artificial vessel network pumped with a blood substitute.

The use of iPPG proves extremely useful for the study of OP models, thanks to the breadth of empirical data it provides, particularly in comparison to point measurement techniques. OP modeling research and its application in PPG bring insights into the physiological phenomena that can be reflected as changes in tissue optical properties and could therefore provide characterization of cardiovascular status. With this approach, effective cardiovascular assessment techniques could be further developed through a better understanding of OP principles and an increasingly informed design of sensing probes for point measurement and noncontact imaging.

\section{ACKNOWLEDGEMENTS}

The authors are grateful to our colleagues at the Photonics Engineering and Health Technology Research Group of Loughborough University for their enormous contributions. The authors also acknowledge the financial support of both the Engineering and Physical Sciences Research Council UK (Optical Platform Grant GR/T25040/01, 2007) and Loughborough University.

\section{CONFLICT OF INTEREST}

The author indicated no potential conflicts of interest.

\section{REFERENCES}

[1] Hertzman AB, Spealman CR, "Observations on the finger volume pulse recorded photoelectrically," American Journal of Physiological Measurement, 1937, 119:334-335.

[2] Nitzan M., Babchenko A, Khanokh B. Landau D, "The variability of the photoplethysmographic signal-a potential method for the evaluation of the autonomic nervous system," Physiological Measurement 1998, 19:93-102.

[3] Challoner AVJ, Ramsay CA, "A photoelectric plethysmograph for the measurement of cutaneous blood flow", Physics in Medicine and Biology, 1974, 19(3):317-328.

[4] Shmada Y, Yoshiya I, Oka N, Mamaguri K, "Effects of multiple scattering and peripheral circulation on arterial oxygen saturation, measured with a pulse-type oximeter" Medical \& Biological Engineering \& Computing, 1984, 22:475-478.

[5] Steinke JM, Shepherd AP, "Role of light scattering in whole blood oximetry", IEEE Transaction on Biomedical Engineering, 1986, 33(3):294-301.

[6] Allen J, Murray A, "Development of a neural network screening aid for diagnosing lower limb peripheral vascular disease from photoelectric plethysmography pulse waveforms", Physiological Measurement, 1993, 14:13-22. 
[7] Allen J, Oates CP, Lees TA, Murray A, "Photoplethysmography detection of lower limb peripheral arterial occlusive disease: a comparison of pulse timing, amplitude and shape characteristics", Physiological Measurement, 2005, 26(5):811-821.

[8] Whiteley MS, Fox AD, Horrocks M, "Photoplethysmography can replace hand-held Doppler in the measurement of ankle/brachial indices", Annals of The Royal College of Surgeons of England, 1998, 809(2):96-98.

[9] Lopez-Beltran EA, Blackshear PL, Finkelstein SM, Cohn JN, "Non-invasive studies of peripheral vascular compliance using a non-occluding photoplethysmographic method", Medical \& Biological Engineering \& Computing, 1998, 36:748-753.

[10] Allen J. Murray A, "Prospective assessment of an artificial neural network for the detection of peripheral vascular disease from lower limb pulse waveforms", Physiological Measurement., 1995, 16:29-38.

[11] Jubran A, "Pulse oximetry", Applied Physiology in Intensive Care Medicine, 2009, 45-48.

[12] Echiadis AS, Crabtree VP, Bence J, Hadjinikolaou L, Alexiou C, Spyt TJ, Hu S, "Non-invasive measurement of peripheral venous oxygen saturation using a new venous oximetry method: evaluation during bypass in heart surgery", Physiological Measurement, 2007, 28(8):897-911.

[13] Vold ML, Aasebø U, Hjalmarsen A, Melbye H, "Predictors of oxygen saturation $\leq 95 \%$ in a crosssectional population based survey”, Respiratory Medicine, 2012, 106(11):1551-1558.

[14] Pak JG, Park KH, "Advanced pulse oximetry system for remote monitoring and management", Journal of Biomedicine and Biotechnology, 2012, 2012:930582.

[15] Moyle J T B 1994 Principles and Practice Series: Pulse Oximetry (London: BMJ Publishing Group).

[16] Agache PG, Dupond AS, "Recent advances in non-invasive assessment of human skin blood flow", Acta dermato-venereologica Supplementum (Stockh), 1994, 185:47-51.

[17] Mannheimer PD, Casciani JR, Fein ME, Nierlich SL, "Wavelength selection for low-saturation pulse oximetry”, IEEE Transactions on Biomedical Engineering, 1997, 44(3):148-158.

[18] Shelley KH, "Photoplethysmography: beyond the calculation of arterial oxygen saturation and heart rate," Anesthesia \& Analgesia. 2007 Dec;105(6 Suppl):S31-6.

[19] O'Connor MJ, Morrow WR, "Pulse oximetry screening for critical congenital heart disease: a review", The Journal of the Arkansas Medical Society, 2012, 109(2):41-3.

[20] Hayes M, Smith P, "Artifact reduction in photoplethysmography", Applied Optics, 1998, 37(31):7437-7446.

[21] Kamat V, "Pulse Oximetry”, Indian Journal of Anesthesia, 2002, 46(4):261.

[22] Bickler PE, Feiner JR, Severinghaus JW, "Effects of skin pigmentation on pulse oximeter accuracy at low saturation", Anesthesiology 2005, 102 (4):715-719.

[23] Hayes MJ, Smith PR, "Quantitative evaluation of photoplethysmographic artefact reduction for pulse oximetry”, Proceeding of SPIE 3, Biomedical Sensors, Fibers, and Optical Delivery Systems, 1998, 3570:138-147.

[24] Subramanian H, Ibey BL, Xu W, Wilson MA, Ericson MN, Coté GL., "Real-time separation of perfusion and oxygenation signals for an implantable sensor using adaptive filtering", IEEE Transactions on Biomedical Engineering, 2005, 52(12):2016-2023.

[25] Cennini G, Arguel J, Akșit K, Leest AV, "Heart rate monitoring via remote photoplethysmography with motion artifacts reduction”, Optics Express, 2010, 18(5):4867-4875.

[26] Poh MZ, McDuff DJ, Picard RW, “Advancements in Noncontact, Multiparameter Physiological Measurements Using a Webcam”, IEEE Transactions on Biomedical Engineering, 2011, 58(1):7-11.

[27] Hu S, Zheng J, Chouliaras V, Summers R, "Feasibility of imaging photoplethysmography", 2008 International Conference on BioMedical Engineering and Informatics, Sanya, China, 2008, 2:72-75.

[28] Zheng J, Hu S, Azorin-Peris V, Echiadis A, Chouliaras V, Summers R, "Remote simultaneous dual wavelength imaging photoplethysmography: a further step towards 3-D mapping of skin blood microcirculation", Proceedings of SPIE, Multimodal Biomedical Imaging III, 2008, 6850, 68500S-1. 
[29] Zheng J, Hu S, Azorin-Peris V, Echiadis, A, Shi P, Chouliaras V, “A remote approach to measure blood perfusion from human face”, Proc. SPIE 7169, Advanced Biomedical and Clinical Diagnostic Systems VII, 2009, 7170-4.

[30] Berndt R.-D, Takenga MC, Kuehn S, Preik P, Stoll N, Thurow K, Kumar M, Weippert M, Rieger A, Stoll R, "A scalable and secure Telematics Platform for the hosting of telemedical applications. Case study of a stress and fitness monitoring", IEEE, 13th International Conference on e-Health Networking, Application and Services, HEALTHCOM, Columbia, USA, 2011, 118-121.

[31] Scully CG, Lee J, Meyer J, Gorbach AM, Granquist-Fraser D, Mendelson Y, Chon KH, "Physiological Parameter Monitoring from Optical Recordings With a Mobile Phone”, IEEE Transactions on Biomedical Engineering, 2012, 59(2):303- 306.

[32] Wieringa FP, Mastik F, van der Steen AF, "Contactless multiple wavelength photoplethysmographic imaging: a first step toward "SpO2 camera" technology", Annals of Biomedical Engineering, 2005, 33(8):1034-1041.

[33] Kamshilin AA, Miridonov S, Teplov V, Saarenheimo R, Nippolainen E, "Photoplethysmographic imaging of high spatial resolution", Biomedical Optics Express, 2011, 2(4):996-1006.

[34] Instant Heart Rate app passes 10M users, Oct. 2011, http://mobihealthnews.com/13600/instant-heartrate-app-passes-10m-users/, accessed Sept. 2012

[35] Philips app measures vitals using iPad camera, Nov. 2011, mobihealthnews.com/14848/philips-appmeasures-vitals-using-ipad-camera/, accessed Sept. 2012.

[36] Azorin-Peris V, Hu S, Smith PR, "A Monte Carlo Platform for the Optical Modelling of Pulse Oximetry", Proceedings of SPIE, Biomedical Applications of Light Scattering, 2007, 64460T.

[37] Mendelson Y, "Pulse oximetry: theory and applications for noninvasive monitoring", Clinical Chemistry, 1992, 38(9):1602-1607.

[38] Tuchin V, Utz S, Yaroslavsky I, "Tissue optics, light distribution and spectroscopy", Optical Engineering, 1994, 33(10):3178-3188.

[39] Ugnell AO, Oberg PA, "The optical properties of the cochlear bone", Medical Engineering \& Physics, 1997, 19(7): 630-636.

[40] Ashley JW, Martin JC, van Gemert, Willem M Star, "Definitions and Overview of Tissue Optics", Optical-Thermal Response of Laser-Irradiated Tissue, SpringerLink, 2011, Part 1, 27-64.

[41] Smith PR, Hayes MJ, "Artefact reduction in Photoplethysmography," International Patent, 1999, WO1999032030.

[42] Krishnan R, Natarajan BB, Warren S, "Two-stage approach for detection and reduction of motion artifacts in photoplethysmographic data", IEEE Transaction on Biomedical Engineering. 2010, 57(8):1867-1876.

[43] Patterson MS, Wilson BC, Wyman DR, "The propagation of optical radiation in tissue I. Models of radiation transport and their application," Lasers in Medical Science. 1991, 6(2):158-168.

[44] Schmitt JM, Zhou GX, Walker EC, Wall RT, "Multilayer model of photon diffusion in skin," Journal of the Optical Society of American A., 1990, 7(11):2141-2153.

[45] Van Gemert MJ, Jacques SL, Sterenborg HJ, Star WM, "Skin optics," IEEE Transaction on Biomedical Engineering. 1989, 36(12):1146-1154.

[46] Richards-Kortum R, Sevick-Muraca E, "Quantitative Optical Spectroscopy for Tissue Diagnosis", Annual Review of Physical Chemistry, 1996, 47:555-606.

[47] Delpy DT, Cope M, van der Zee P, Arridge S, Wray S, Wyatt J, "Estimation of optical pathlength through tissue from direct time of flight measurement", Physics in Medicine and Biology, 1988, 33(12):1433-1442.

[48] Hiraoka M, Firbank M, Essenpreis M, Cope M, Arridge SR, van der Zee P, Delpy DT, “A Monte Carlo investigation of optical pathlength in inhomogeneous tissue and its application to near-infrared spectroscopy", Physics in Medicine and Biology, 1993, 38:1859-1876.

[49] Konig V, Huch R, Huch A, "Reflectance pulse oximetry-principles and obstetric application in the zurich system," Journal of Clinical Monitoring, 1998, 14:403-412. 
[50] Meglinskin IV, Matcher SJ, "Computer simulation of the skin reflectance spectra," Computer Methods and Programs in Biomedicine, 2003, 70:179-186.

[51] Reuss J, "Multilayer modelling of reflectance pulse oximetry," IEEE Transaction on Biomedical Engineering, 2005, 52:153-160.

[52] Azorin-Peris V, Opto-Physiological Modeling of Pulse Oximetry, PhD thesis, Loughborough University, 2008.

[53] Takatani S, Davies C, Sakakibara N, Zurick A, Kraenzler E, Golding LR, Noon GP, Nose Y, DeBakey ME., "Experimental and clinical evaluation of a noninvasive reflectance pulse oximeter sensor", Journal of Clinical Monitoring, 1992, 8(4):257-266.

[54] Nickell S, Hermann M, Essenpreis M, Farrell TJ, Kramer U, Patterson MS, "Anisotropy of light propagation in human skin," Physics in Medicine and Biology. 2000, 45(10):2873-2886.

[55] Enejder AM, Swartling J, Aruna P, Andersson-Engels S, "Influence of cell shape and aggregate formation on the optical properties of flowing whole blood," Applied Optics. 2003, 42(7):1384-1394.

[56] Bolin FP, Preuss LE, Taylor RC, Ference RJ, "Refractive index of some mammalian tissues using a fiber optic cladding method," Applied Optics, 1989, 28:2297-2303.

[57] Schmitt JM, "Simple photon diffusion analysis of the effects of multiple scattering on pulse oximetry," IEEE Transaction on Biomedical Engineering, 1991, 38(12):1194-1203.

[58] Bashkatov AN, Genina EA, Tuchin VV, "Optical properties of skin, subcutaneous and muscle tissues: a review," Journal of Innovative Optical Health Sciences, 2011, 4(1): 9-38.

[59] Ballerini L, Fisher RB, Aldridge B, Rees, J "A Color and Texture Based Hierarchical K-NN Approach to the Classification of Non-melanoma Skin Lesions", Color Medical Image Analysis, Lecture Notes in Computational Vision and Biomechanics, 2013, 6:63-86.

[60] Tulppo MP, Makikallio T H, Takala T E, Seppanen T, Huikuri HV, "Quantitative beat-to-beat analysis of heart rate dynamics during exercise," The American Journal of Physiology, 1996, 271:H244-252.

[61] Reddy KA, George B, Kumar VJ, "Use of Fourier Series Analysis for Motion Artifact Reduction and Data Compression of Photoplethysmographic Signals", IEEE Transactions on Instrumentation and Measurements, 2009, 58(5):1706-1711.

[62] Sun Y, Hu S, Azorin-Peris V, Greenwald S, Chambers J, Zhu Y., "Motion-compensated noncontact imaging photoplethysmography to monitor cardiorespiratory status during exercise," Journal of Biomedical Optics, 2011, Jul;16(7):077010.

[63] Azorin-Peris V, Hu S, "Validation of a Monte Carlo platform for the optical modeling of pulse oximetry", Journal of Physics: Conference Series, 2007, 85:012027

[64] Zheng J, Opto-physiological Modeling of Imaging Photoplethysmography, Ph.D. Thesis, Loughborough University, UK, 2010

[65] Rajan K, Patnaik LM, "CBP and ART image reconstruction algorithms on media and DSP processors", Microprocessors and Microsystems, 2001, 25(5):233-238.

[66] Dandekar O, Shekhar R, "FPGA-Accelerated Deformable Image Registration for Improved TargetDelineation During CT-Guided Interventions", IEEE Transactions on Biomedical Circuits and Systems, 2007, 1(2):116-127.

[67] Chouliaras VA, Nunez JL, Mulvaney DJ, Rovati F, Alfonso D, "A Multi-standard Video accelerator based on a vector architecture", IEEE Transactions on Consumer Electronics, 2005, 51(1):160-167.

[68] Archer CB, Functions of the Skin, Rook's Textbook of Dermatology, edited by Tony Burns, Stephen Breathnach, Neil Cox, Christopher Griffiths, Wiley-Blackwell, 2010.

[69] Stevens D, Chouliaras, VA, Azorin-Peris V, Zheng J, Echiadis A, Hu S, "BioThreads: A Novel VLIWBased Chip Multiprocessor for Accelerating Biomedical Image Processing Applications”, IEEE Transactions on Biomedical Circuits and Systems, 2012, 6(3):257- 268. 


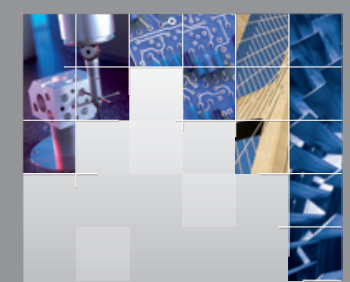

\section{Enfincering}
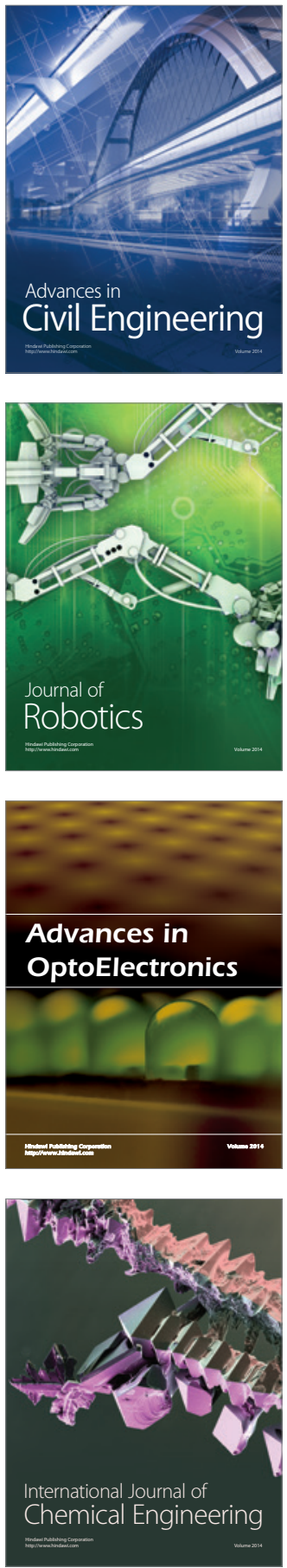

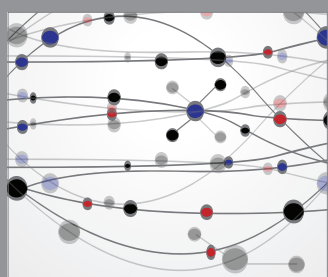

The Scientific World Journal

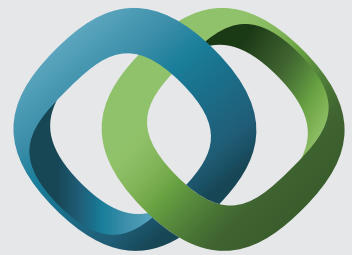

\section{Hindawi}

Submit your manuscripts at

http://www.hindawi.com
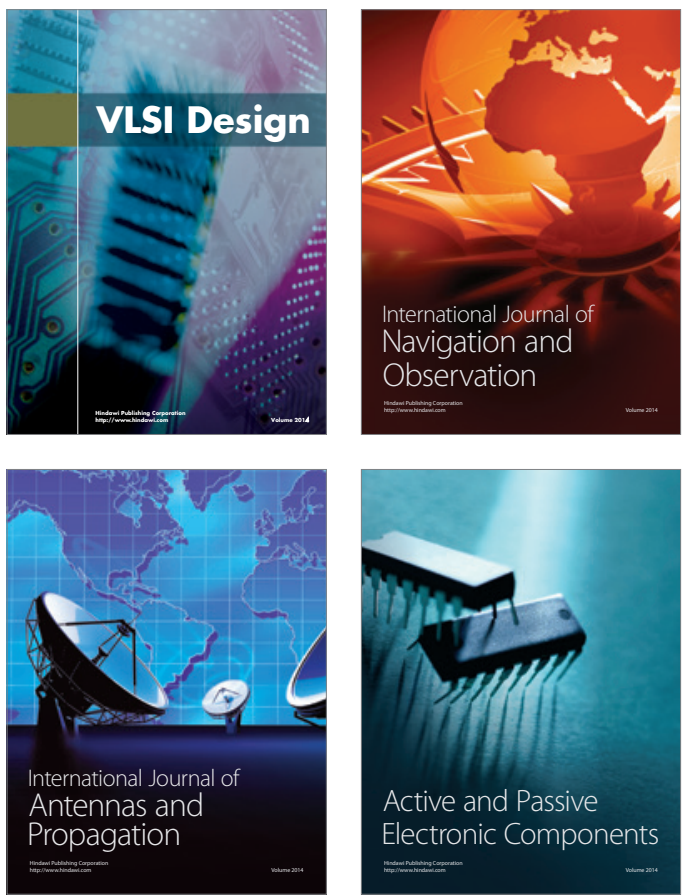
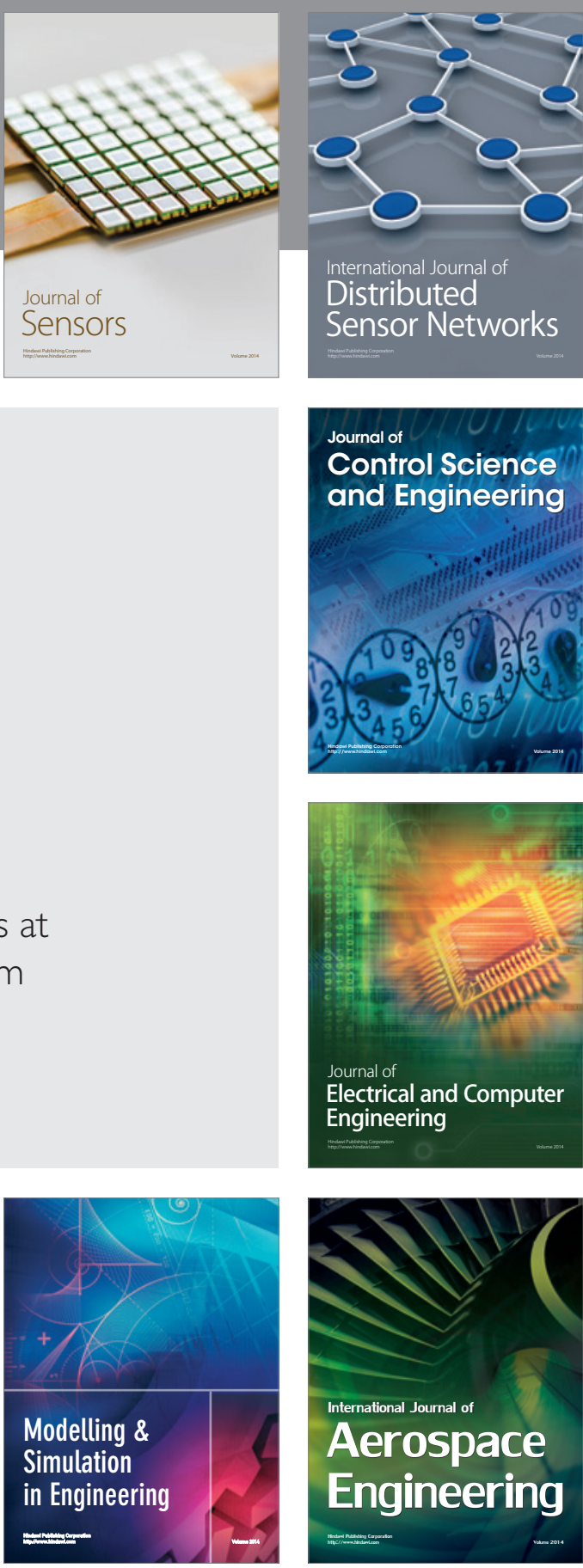

International Journal of

Distributed

Sensor Networks

Journal of

Control Science

and Engineering
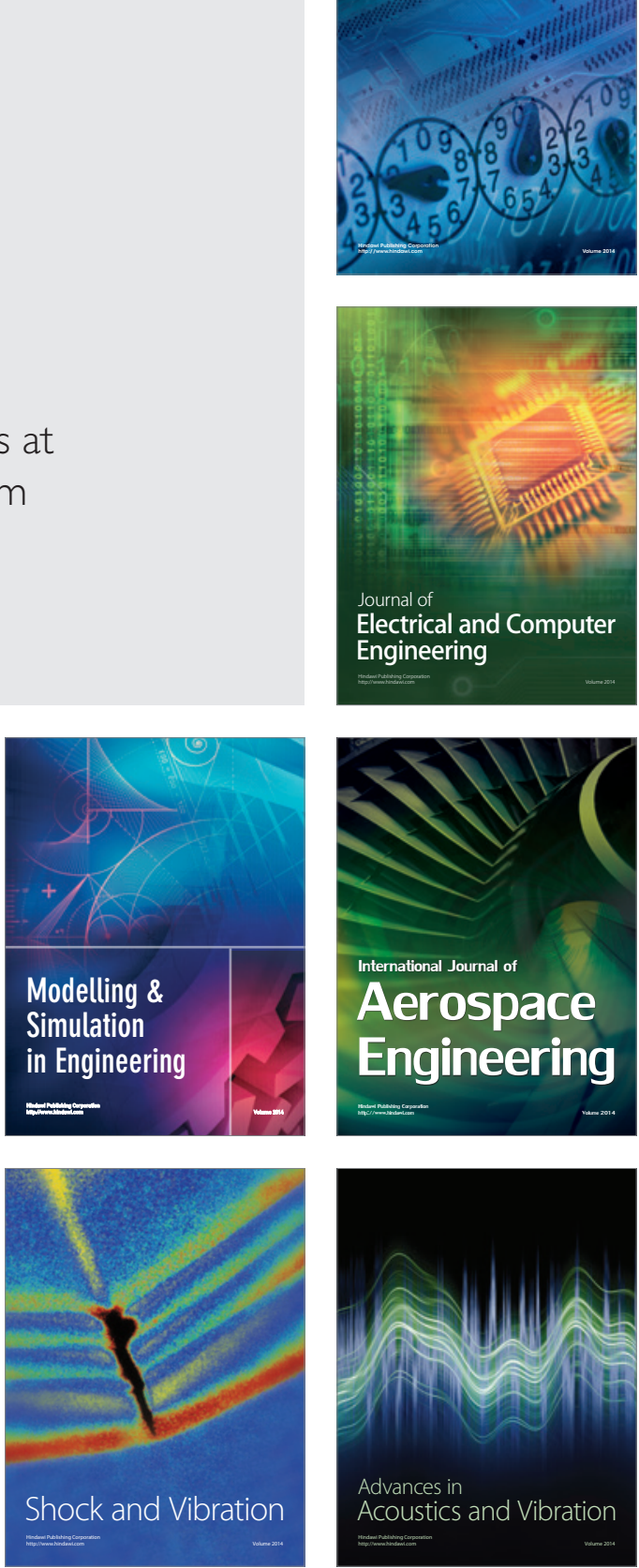